\title{
Estilo de vida dos universitários do curso de Nutrição
}

\author{
Lifestyle of the Nutrition courses university
}

Edilainy Caroliny de Souza', Rayane Mendes dos Santos', Lucinéia de Pinho²

${ }^{7}$ Nutricionista - Faculdade de Saúde Ibituruna - FASI

${ }^{2}$ Nutricionista, Doutora em Ciências da Saúde - Universidade Estadual de Montes Claros Unimontes/Faculdade de Saúde Ibituruna - FASI

E-mail para contato: Lucinéia de Pinho - Iucineiapinho@hotmail.com

\section{Resumo}

Objetivo: avaliar o estilo de vida de universitários do curso de Nutrição. Metodologia: trata-se de uma pesquisa quantitativa, com abordagem transversal e descritiva realizada com 108 acadêmicos matriculados e frequentes no curso de Nutrição de duas faculdades privadas, na cidade de Montes Claros, Minas Gerais. Para a coleta de dados foi aplicado o questionário validado Estilo de Vida Fantástico. O estudo teve a aprovação do Comitê de Ética e Pesquisa da SOEBRAS. Resultados: entre os universitários a maioria (44\%) apresentou um bom estilo de vida. Ao associar as classificações "bom", "muito bom" e "excelente", 92 universitários (85\%) atingiram estilo de vida satisfatório, 16 deles (15\%) obtiveram classificação regular e nenhum dos avaliados obteve pontuação inferior a 35 pontos, medida limítrofe que indica necessidade de melhora. Conclusão: os acadêmicos possuem um estilo de vida adequado. No entanto, os indicadores do estilo de vida apontam a necessidade de melhoras no que diz respeito aos aspectos relacionados principalmente ao componente nutrição, introspecção e atividade física.

Palavras-chave: Estilo de vida. Universitários. Fatores de risco.

\begin{abstract}
Objective: to evaluate the lifestyle of university students of the Nutrition course. Methodology: This is a quantitative research, with a cross-sectional and descriptive approach carried out with 108 students enrolled and frequent in the Nutrition course of two private colleges, in the city of Montes Claros, Minas Gerais. For the data collection, the validated questionnaire was used. The study was approved by the Ethics and Research Committee of SOEBRAS. Results: the majority of university students (44\%) have a good lifestyle. By associating the "good", "very good" and "excellent" classifications, 92 university students (85\%) achieved a satisfactory lifestyle, 16 of them (15\%) obtained a regular classification and none of the evaluated ones scored below 35 points, Measure that indicates need for improvement. Conclusion: academics have an adequate lifestyle. However, lifestyle indicators point to the need for improvements in aspects related mainly to the nutrition, introspection and physical activity component.
\end{abstract}

Keywords: Lifestyle. University students. Risk factors. 


\section{INTRODUÇÃO}

O estilo de vida pode ser definido como uma condição de vida fundamentada em práticas identificáveis de hábitos conscientes, os quais são determinados pela interação de papéis entre as características pessoais, relações sociais e os padrões ou níveis de vida socioeconômicos e ambientais. Dessa forma, o estilo de vida é capaz de determinar um resultado profundo na saúde dos indivíduos ${ }^{1}$.

Há aspectos do estilo de vida que são capazes de atingir de maneira negativa a saúde, e para os quais se pode ter controle, denominados fatores negativos modificáveis como o tabagismo, o alcoolismo, drogas e falta de atividade física. Existem ainda os fatores positivos que se praticados de forma adequada, colaboram também para um estilo de vida saudável, como a alimentação, exercícios físicos e comportamento preventivo (uso de preservativos, cinto de segurança etc). Os fatores positivos são fundamentais para preservar as condições de saúde pretendidas, uma vez que evitam as doenças e os danos não transmissíveis à saúde, como cardiopatias, hipertensão arterial e câncer ${ }^{2}$.

São crescentes as evidências de que a maneira como se vive é um fator diferencial para a saúde e a qualidade de vida nos dias atuais, independente da idade ou ambiente social e econômico ${ }^{3}$. Diante disso, em todas as etapas da vida é necessário indagar a presença de situações possíveis de provocar doenças no futuro. O público universitário é um grupo vulnerável, uma vez que o acadêmico passa por variadas mudanças que alteram o seu estilo de vida e isso pode colaborar para o surgimento de múltiplos fatores de risco para as doenças crônicas não transmissíveis ${ }^{3,4}$.

A população de jovens e adultos progressivamente sofre doenças resultantes do estilo de vida e ligadas aos fatores de risco modificáveis, que poderiam ser reduzidas pela prática de uma vida saudável, já que o estilo de vida pode ser estabelecido durante a juventude, antes da vida adulta e é capaz de influenciar nas próximas fases da vida 5, 6 .

Diversos fatores do estilo de vida podem ser incorporados no dia a dia dos universitários na fase da passagem do ensino médio para o ensino superior. Isso torna os acadêmicos um grupo susceptível à adesão de comportamentos adequados e/ou inadequados para a saúde7.

A investigação do estilo de vida de estudantes universitários é essencial, principalmente no que diz respeito aos aspectos e hábitos do dia a dia que podem ser a causa de doenças crônicas não transmissíveis em um futuro próximo. Dessa forma, 
o presente estudo teve como objetivo avaliar o estilo de vida dos universitários do curso de Nutrição da cidade de Montes Claros, Minas Gerais.

\section{MÉTODOS}

O presente estudo se caracterizou como uma pesquisa quantitativa, com abordagem transversal e descritiva, realizado em duas faculdades privadas de Montes Claros. Participaram da pesquisa acadêmicos que cursavam do $1^{\circ}$ ao $8^{\circ}$ período do curso de Nutrição. Foram incluídos no estudo todos os universitários matriculados e frequentes no curso das duas instituições e que aceitaram participar de forma voluntária da pesquisa, mediante a entrega do Termo de Consentimento Livre e Esclarecido devidamente assinado. Foram excluídos aqueles que não compareceram para a coleta dos dados após duas tentativas, apresentaram alguma condição física que impossibilite responder ao questionário auto administrado ou não responderam corretamente o questionário.

\section{Instrumentos}

Para obtenção dos indicadores socioeconômicos e demográficos foi aplicado um questionário semiestruturado elaborado pelos pesquisadores. Fez-se a aplicação ainda de um segundo questionário. Este, por sua vez é validado, avalia o estilo de vida e já foi empregado em outros estudos com estudantes no Brasil: Questionário Estilo de Vida Fantástico. O instrumento é padronizado com 25 questões fechadas que analisam nove domínios: família e amigos, atividade física, nutrição, tabaco e tóxicos, álcool, sono, cinto de segurança, estresse e sexo seguro, tipo de comportamento, introspecção e trabalho. As perguntas estão organizadas na forma de escala Likert, sendo que 23 possuem cinco possibilidades de resposta e duas são dicotômicas. A numeração das questões é realizada por pontos, sendo que cada coluna possui um peso distinto variando de 0 a 4 pontos. As perguntas que só possuem duas alternativas pontuam: zero e 4 respectivamente. A soma de todos os pontos permite classificar os indivíduos em cinco categorias: "Excelente" (85 a 100 pontos), "Muito bom" (70 a 84 pontos), "Bom" (55 a 69 pontos), "Regular" (35 a 54 pontos) e "Necessita melhorar" (o a 34 pontos) ${ }^{8}$.

Os questionários foram aplicados pelos próprios pesquisadores no primeiro semestre de 2017 entre os meses de março e abril. Foi verificado junto ao professor o melhor horário, cerca de 10 minutos, para que a proposta da pesquisa fosse apresentada aos participantes, e os objetivos desta, fossem esclarecidos. Os 
acadêmicos assinaram o Termo de Consentimento Livre e Esclarecido, receberam os questionários e foram orientados a preenchê-los de forma extracurricular e devolvê-los no dia seguinte devidamente respondidos.

Os dados coletados foram planilhados no Microsoft Excel 2010 e posteriormente foram feitas análises de frequência.

O projeto foi aprovado pelo Comitê de Ética e Pesquisa da Associação Educativa do Brasil-SOEBRAS, sob o registro CAAE 6321/16.8.0000.5141/2017.

\section{RESULTADOS}

\section{Caracterização sociodemográfica}

Participaram desse estudo 108 estudantes do curso de Nutrição de duas faculdades privadas da cidade de Montes Claros, Minas Gerais.

A amostra foi predominantemente composta por universitários do sexo feminino $(88,8 \%)$, com idade superior a 20 anos $(51 \%)$, sem companheiro $(85,2 \%)$, não trabalham de forma remunerada $(54,6 \%)$ e com escolaridade materna maior que oito anos (65,7\%) (Tabela 1).

Tabela 1. Distribuição da amostra em relação às características demográficas e socioeconômicas. Montes Claros, Minas Gerais, Brasil, 2017 ( $n=108)$.

\begin{tabular}{l|c|c}
\hline Variáveis & N & $\%$ \\
\hline Sexo & 12 & 11,2 \\
Masculino & 96 & 88,8 \\
$\begin{array}{l}\text { Feminino } \\
\text { Idade }\end{array}$ & 53 & 49 \\
$\leq 20$ anos & 55 & 51 \\
> 20 anos & & \\
Situação conjugal & 92 & 85,2 \\
Sem companheiro & 16 & 14,8 \\
Com companheiro & & \\
Trabalho remunerado & 59 & 54,6 \\
Não & 49 & 45,4 \\
Sim & 71 & 65,7 \\
Escolaridade da mãe & 37 & 34,3 \\
$>8$ anos & & \\
S 8 anos & & \\
\end{tabular}

\section{Estilo de vida fantástico}

Foi possível observar que os universitários de uma forma geral, apresentaram um estilo de vida de bom a muito bom. O estudo evidenciou que os pontos alcançados pelo questionário foram próximos dos 70 pontos. 
Entre os universitários a maioria (44\%) apresentou um bom estilo de vida. Ampliando-se essa análise, pôde-se observar ainda que ao agrupar as classificações "bom", "muito bom" e "excelente", obtém-se um total de 92 universitários (85\%) que apresentam um estilo de vida satisfatório. É importante destacar que nenhum dos avaliados obteve pontuação inferior a 35 pontos, medida limítrofe que indica necessidade de melhora. Porém, 16 deles (15\%) obtiveram classificação regular (Tabela 2).

Tabela 2 - Classificação do estilo de vida dos universitários do curso de Nutrição Montes Claros, Minas Gerais, Brasil

\begin{tabular}{l|c|c}
\hline Categorias (n=108) & Pontos & n (\%) \\
\hline Excelente & $85-100$ & $6(5,55 \%)$ \\
Muito bom & $70-84$ & $38(35,18 \%)$ \\
Bom & $55-69$ & $48(44,44 \%)$ \\
Regular & $35-54$ & $16(14,81 \%)$ \\
\hline
\end{tabular}

Conforme a tabela 3 observaram-se elevados índices de hábitos positivos no que se refere ao relacionamento com a família e os amigos $(67,6 \%)$.

No que diz respeito à atividade física, verificaram-se elevados índices de hábitos negativos. Cerca de $91,7 \%$ praticam uma quantidade mínima de atividade física diariamente, indicando comportamentos sedentários desses universitários.

Quanto ao aspecto nutrição, a maioria apresenta hábitos negativos, uma vez que 87\% dos universitários analisados consomem uma alimentação inadequada.

Em referência ao álcool, tabaco e tóxicos, a maior parte dos universitários considerados, apresentou elevados índices de hábitos positivos, representando $56,5 \%$ e $63,9 \%$, respectivamente, o que aponta um baixo consumo desses produtos.

Não eram adequados em relação ao sono, uso de cinto de segurança, estresse e sexo seguro, $84,3 \%$ dos universitários.

Foi possível observar ainda que 78,7\% apresentam uma relação inadequada quanto ao tipo de comportamento diário, além de problemas de introspecção $78 \%$ e cerca de $53,7 \%$ não estavam satisfeitos com o trabalho que realizavam.

Tabela 3 - Média da pontuação obtida nos domínios do questionário Estilo de Vida Fantástico dos universitários do curso de Nutrição Montes Claros, Minas Gerais, Brasil.

\begin{tabular}{l|c|c}
\hline Domínios & $\mathbf{n}$ & $\%$ \\
\hline Família e amigos & 73 & $67,6 \%$ \\
Satisfeito & 35 & $32,4 \%$ \\
Insatisfeito & Continua
\end{tabular}


Continuação da tabela 3

\begin{tabular}{|c|c|c|}
\hline Domínios & $\mathbf{n}$ & $\%$ \\
\hline \multicolumn{3}{|c|}{ Atividade física } \\
\hline Ativo & 9 & $8,3 \%$ \\
\hline Inativo & 99 & $91,7 \%$ \\
\hline \multicolumn{3}{|l|}{ Nutrição } \\
\hline Adequada & 14 & $13 \%$ \\
\hline Inadequada & 94 & $87 \%$ \\
\hline \multicolumn{3}{|c|}{ Tabaco e tóxicos } \\
\hline Não Consome & 69 & $63,9 \%$ \\
\hline Consome & 39 & $36,1 \%$ \\
\hline \multicolumn{3}{|l|}{ Álcool } \\
\hline Adequado & 61 & $56,5 \%$ \\
\hline Inadequado & 47 & $43,5 \%$ \\
\hline \multicolumn{3}{|c|}{ Sono, CS, estresse e SS } \\
\hline Adequado & 38 & $35,2 \%$ \\
\hline Inadequado & 70 & $64,8 \%$ \\
\hline \multicolumn{3}{|c|}{ Tipo de comportamento } \\
\hline Adequado & 23 & $21,3 \%$ \\
\hline Inadequado & 85 & $78,7 \%$ \\
\hline \multicolumn{3}{|l|}{ Introspecção } \\
\hline Adequada & 27 & $25 \%$ \\
\hline Inadequada & 81 & $78 \%$ \\
\hline \multicolumn{3}{|c|}{ Trabalho (função) } \\
\hline Satisfeito & 50 & $46,3 \%$ \\
\hline Insatisfeito & 58 & $53,7 \%$ \\
\hline
\end{tabular}

CS: cinto de segurança; SS: sexo seguro

\section{DISCUSSÃO}

Este estudo realizado em duas instituições privadas de Montes Claros evidenciou que a maioria dos estudantes de Nutrição possuem um estilo de vida satisfatório. Este resultado é similar ao encontrado em um estudo que avaliou estudantes universitários ( $n=672$ ) utilizando o mesmo questionário, com média de idade de 22,5 anos, e constataram que $42,49 \%$ dos participantes foram classificados com estilo de vida fantástico bom, ou muito bom9.

Apesar dos estudantes da área da saúde terem como principal objetivo profissional manter ou melhorar a qualidade de vida, principalmente no que diz respeito à alimentação, muitos possuem maus hábitos de vida, como a própria alimentação inadequada e baixos níveis de atividade física. A junção destes dois hábitos negativos tem sido apontada como a principal causa para o surgimento de uma série de 
doenças, sobretudo as doenças crônicas não transmissíveis como o câncer, infarto do miocárdio, hipertensão, acidente vascular cerebral, além da obesidade, do sobrepeso e outras patologias. Há a necessidade de melhoria no manejo da qualidade de vida dos universitários da área de saúde por meio de medidas de incentivo para a promoção de saúde e prevenção de doenças ${ }^{10}$.

No que diz respeito ao nível de atividade física e alimentação, no estudo prevaleceu universitários que afirmaram possuírem um baixo nível de atividade física, representando $91,7 \%$ de todos os universitários investigados, e no domínio nutrição, apenas $13 \%$ consomem uma alimentação adequada.

Os hábitos saudáveis não atingem níveis ideais nessa população, sendo a saúde negligenciada em detrimento das atividades acadêmicas ${ }^{11}$. Este estudo reforça a ideia de que mesmo com uma rotina intensa de afazeres, é preciso incluir a prática de atividade física e alimentação saudável no dia a dia, priorizando um estilo de vida mais saudável, com treinamento para o manejo de tempo, permitindo que conciliem as atividades acadêmicas com o cuidado da saúde.

Belem et $\mathrm{al}^{12}$, avaliando universitários em diferentes faixas de idade mostraram que há diferenças entre os grupos. Evidenciou-se que os universitários que apresentaram um estilo de vida negativo, eram os mais jovens, com idade até 24 anos. Sugerem que a instituição de ensino ofereça no próprio ambiente do campus já no ingresso na instituição atividades diversas que favoreçam a adoção de um estilo de vida saudável.

O domínio nutrição apresentou uma média considerada baixa, principalmente se tratando de uma pesquisa feita com acadêmicos de Nutrição. Os resultados do presente estudo são semelhantes àqueles observados por Oliveira et $\mathrm{al}^{13}$, que mostram a inadequação na ingestão alimentar de universitários matriculados no curso de Nutrição em Sergipe. Observou-se que 53\% dos estudantes referiram o consumo semanal de doces, assim como $14 \%$ de fast food. Contrariando esta ideia, Souza et $\mathrm{al}^{14}$ afirma que ao avaliar estudantes do curso de Educação Física e Fisioterapia, foi observado que a maioria dos pesquisados consome pouco fast foods apesar do pouco tempo dedicado às refeições. Este resultado é justificado pelo fato dos universitários morarem com os pais e aumentar a inclusão na alimentação as verduras, frutas, hortaliças e grãos nas refeições.

Em um estudo conduzido por Siqueira et $\mathrm{al}^{15}$ entre profissionais de saúde observouse que a ausência do perfil de saúde foi associada com excesso de peso. No presente 
estudo a presença de comportamentos de saúde inadequados em futuros profissionais da saúde pode levar a um quadro similar e, ainda afetar as atitudes dos pacientes na motivação para fazer mudanças no estilo de vida.

No que se refere ao cigarro, outras drogas e o álcool os achados deste estudo corroboram com os encontrados por Barbosa et $\mathrm{al}^{16}$ em uma pesquisa feita com acadêmicos de medicina, onde a pontuação que avalia estes três domínios foi alta, o que indica uma boa qualidade de vida neste aspecto.

O domínio família e amigos obteve uma boa pontuação em grande parte dos questionários respondidos. Pereira et $\mathrm{al}^{17}$ evidenciaram que fatores protetivos como o relacionamento familiar e de amizade são importantes e fazem diferença para a resolução de problemas na adultez emergente. Esses fatores se mostraram essenciais para os indivíduos não apresentarem ideação suicida como uma opção para lidar com as situações de desafio da vida. É possível perceber que boas relações afetivas colaboram para um melhor estilo de vida global.

Para o componente comportamento preventivo, foi observado um mau hábito do estilo de vida dos universitários. A falta de comportamento preventivo, pode tornar os universitários um grupo vulnerável a riscos significativos com relação a sua saúde $^{18}$.

Para este aspecto observaram-se índices inferiores. Isto contradiz o observado por Nogueira et $\mathrm{al}^{19}$ que avaliaram o estilo de vida dos acadêmicos de Fisioterapia e Educação física, e demostram que de modo geral, não se observaram elevados índices negativos ao ponto de ser preocupante, uma vez que os alunos têm conhecimentos acerca dos relacionamentos sociais que são fatores que podem prejudicar o rendimento. Conforme uma pesquisa feita por Soares et $\mathrm{al}^{20}$, foi identificado que os alunos que apresentam comportamentos sociais acadêmicos desadaptativos também apresentam déficits em habilidades sociais dificultando sua adaptação acadêmica e que, habilidades socialmente aceitáveis estão associadas não só a varáveis pessoais, mas também às curriculares e institucionais.

Já a pesquisa feita por Barbosa et $\mathrm{al}^{16}$, com estudantes de medicina, vai ao encontro do resultado obtido na presente análise, uma vez que eles apresentaram pontuações baixas nos domínios: comportamento, introspecção, trabalho, sono, cinto de segurança, estresse e sexo seguro e nutrição. 
Destaca-se que o pequeno tamanho amostral foi considerado como um fator limitante do presente estudo, o que pode ter contribuído para que algumas correlações não tenham sido estatisticamente significativas.

\section{CONCLUSÃO}

Conclui-se que a maioria dos acadêmicos de Nutrição avaliados possuem um estilo de vida adequado. No entanto, os indicadores do estilo de vida apontam a necessidade de melhoras no que diz respeito aos aspectos relacionados principalmente ao componente nutrição, introspecção e também a atividade física. Um bom hábito alimentar agregado ao exercício físico é capaz de influenciar diretamente em alguns fatores que causam diversas patologias, inclusive reduzir as chances de adquirir sobrepeso.

Os dados deste estudo podem contribuir para a criação e otimização de projetos dentro das instituições de ensino superior incentivando a socialização entre os alunos, a ingestão de uma alimentação de qualidade e a realização de atividade física.

\section{REFERÊNCIAS}

1. World Health Organization (WHO). The World health report 1998: Life in the 21st century: a vision for all. Genevra: WHO; 1998.

2. Silva DAS, Pereira IMM, Almeida MB, Silva RJS, Oliveira ACC. Estilo de vida de acadêmicos de educação física de uma universidade pública do estado de Sergipe, Brasil. Rev Bras Ciênc Esporte. 2012;34(1):53-67.

3. Nahas MV, Barros MVG, Francalacci VL. O Pentáculo do BemEstar. Base conceitual para avaliação do estilo de vida de indivíduos ou grupos. Rev Bras Ativ Fís Saúde. 2000; 5: 48- 59.

4. Meira MDD, Abdala GA, Teixeira CA, Ninahuaman MFML, Moraes MCL, Salgueiro MMHAO. Perfil do estilo de vida de adultos da zona sul de São Paulo. LyfeStyle Journal. 2015;2(2):67-82.

5. Silva DAS, Quadros TMB, Gordia AP, Petroski EL. Associação do sobrepeso com variáveis sócio demográficas e estilo de vida em universitários. Rev Saúde Col.2011;16(11):4473-4479.

6. Lima ACS, Araújo MFM, Freitas RWJF, Zanetti ML, Almeida PC, Damasceno MMC. Fatores de risco para diabetes mellitus tipo 2 em universitários: associação com variáveis sociodemográficas. Rev Latino-am Enfermagem.2014;22(3);484-490.

7. Rovida TAS, Sumida DH, Santos AS, Moimaz SAS, Garbin CAS. Estresse e o estilo de vida dos acadêmicos ingressantes em um curso de graduação em Odontologia. Rev ABENO. 2015; 15 (3):26-34. 
8. Anez CRR, Reis RS, Petroski EL. Versão brasileira do questionário "estilo de vida fantástico": tradução e validação para adultos jovens. Arq Bras Cardiol.2008;91(2):102-9.

9. Bomfim RA, Mafra MAS, Gharib Iad MS, De-Carli AD, Zafalon E. Fatores associados ao estilo de vida fantástico de universitários brasileiros - uma análise multinível. Rev CEFAC. 2017; 19(5): 601-10.

10. Tassini CC, Val GR, Candido SS, Bachur CK. Avaliação do Estilo de Vida em Discentes Universitários da Área da Saúde através do Questionário Fantástico. Int J Cardiovasc Sci. 2017; 30(2): 117-22.

11. Bührer BE, Tomiyoshi AC, Furtado MD, Nishida FS. Análise da Qualidade e Estilo de Vida entre Acadêmicos de Medicina de uma Instituição do Norte do Paraná. Revista Brasileira de Educação Médica. 2019; 43(1), 39-46.

12. Belem IC, Dias DAC, Both Jorge. Diferentes comportamentos do estilo de vida de universitários do curso de educação física. Pensar en Movimiento. 2019; 17(2): 183-97.

13. Oliveira JS, Santos DO, Rodrigues, SJM, Oliveira CC, Souza AL.C. Avaliação do perfil sociodemográfico, nutricional e alimentar de estudantes de nutrição de uma universidade pública em Lagarto-SE. RASBRAN. 2018; 8(2), 37-42.

14. Souza JV, Bastos TPF, Oliveira MFA. Perfil dos alunos universitários dos cursos de educação física e fisioterapia em relação à alimentação e a atividade física. Rev Práxis. 2014; 11:104-13.

15. Siqueira FV, Reis DS, Souza RAL, Pinho S, Pinho L. Excesso de peso e fatores associados entre profissionais de saúde da Estratégia Saúde da Família. Cad Saúde Colet. 2019; 27(2): 138-45.

16. Barbosa RR, Martins MCG, Carmo FPT, Jacques TM, Serpa RG, Calil OA, Barbosa LFM. Estudo sobre Estilos de Vida e Níveis de Estresse em Estudantes de Medicina. Int J Cardiovasc Sci.2015;28(4):313-19.

17. Pereira AS, Willhelm AR, Koller SH, Almeida RMM. Fatores de risco e proteção para tentativa de suicídio na adultez emergente. Ciênc Saúde Coletiva. 2018; 23 (11): 3767-77.

18. Lima CAG, Maia MFM, Magalhães TA, Oliveira LMM, Reis VMCP, Brito MFSF, Pinho L, Fagundes MS. Prevalência e fatores associados a comportamentos de risco à saúde em universitários no norte de Minas Gerais. Cad Saúde Colet. 2017; 25(2): 183-91.

19. Nogueira AP, Rossato, M, Soares AA, Bezerra ES, Moraes MS. O estilo de vida dos universitários da Faculdade de Educação Física e Fisioterapia da UFAM. Rev Port Ciên Despo. 2014,689-701.

20. Soares AB, Porto AM, Lima CA, Gomes C, Rodrigues DA, Zanoteli R, Santos ZA, Fernandes A, Medeiros H. Vivências, Habilidades Sociais e Comportamentos Sociais de Universitários. Psic: Teor e Pesq. 2019; 34, e34311.

\footnotetext{
Submissão: $17 / 03 / 2020$
}

Aprovação: $25 / 11 / 2020$ 